\title{
Electromagnetic Metrology for Nano- Electromechanical Systems
}

\author{
Ling $\mathrm{Hao}^{\circledR}$, John C. Gallop, and Jie Chen, Member, IEEE
}

\begin{abstract}
This paper outlines how demands on electromagnetic metrology have developed over recent years with the continuing reduction in the typical length scale of electronic components and circuits. In addition, novel materials, especially 2-D self-supporting structures such as graphene, have properties quite unlike the conventional systems. At the nanoscale radical changes occur in techniques required and even in the physical quantities measured. This paper focusses on the example of a near-field scanning microwave microscope and its application to nano-electromechanical system resonators to illustrate these changes and challenge.
\end{abstract}

Index Terms-2-D materials, electrical conductivity, graphene, microwave, nano-electromechanical system (NEMS) resonator, nanoscale electromagnetic metrology, noncontacting scanning microwave microscope (NSMM), SMM.

\section{INTRODUCTION}

I MMENSE strides have been taken over the past 50 years in replacing artifact definitions of the SI-base units by those based on fundamental constants [1]. It is important to recognize that the development of metrology relating to the SI is not "complete" once the base units are all defined in this way. New physics, new materials, and new technologies will always emerge and will require developments and extensions to the SI. A current significant development involves the extension of precise metrology towards the nanoscale. Developments in this area not only require novel techniques but it must also be acknowledged that as the length scale reduces the behavior of the system moves away from the classical concept of an ensemble of particles toward an approach based on a finite number of entities, whether they be molecules, atoms, photons, spins, or even phonons. Ultimately metrology at or even below the nanoscale is concerned with the measurement of single entities and the inevitable quantum mechanical issues which arise. However, long before the single entity level or true quantum mechanical behavior is attained, many other challenges to classical electrical metrology arise as the length scale decreases [2]. Some key issues are outlined in Section II.

Manuscript received July 7, 2018; revised October 8, 2018; accepted October 16, 2018. This work was supported in part by the U.K. NMS Programme and in part by EPSRC. The Associate Editor coordinating the review process was Dr. Sophie Djordjevic. (Corresponding author: Ling Hao.)

L. Hao and J. C. Gallop are with the National Physical Laboratory, Teddington TW11 0LW, U.K. (e-mail: ling.hao@npl.co.uk; john.gallop@npl.co.uk).

J. Chen is with the Department of Mechanical and Aerospace Engineering, Brunel University, Uxbridge UB8 3PH, U.K. (e-mail: jie.chen@brunel.ac.uk).

Color versions of one or more of the figures in this paper are available online at http://ieeexplore.ieee.org.

Digital Object Identifier 10.1109/TIM.2018.2879068
As an example, Section VI focus on measurement issues related to scanned microwave microscopy (SMM) [3] applied to nano-electromechanical system (NEMS) resonators [4], [5].

\section{CONTACTs, Interfaces, AND Dimensionality}

Conventional electromagnetic measurements involve attaching conducting leads to a specimen under test (SUT) and then applying stationary or time-varying currents or voltages to these leads while measuring the response of the SUT via other connections. Measurement of conductivity, resistance, capacitance, inductance, and magnetization can all be carried out in this way for samples of millimeter size and larger, at relatively low frequencies (e.g., $<1 \mathrm{GHz}$ ).

With the development of submicrometer scale lithography new techniques for electromagnetic metrology were needed. Increasingly, nonlinear properties of the SUT were required to be measured, due to growing importance of semiconductors. Further developments toward ever smaller lengths have led to, and enabled, nanoscale electromagnetic devices. Particularly significant have been the improvements in electron beam lithography, focused ion beam milling, and scanned probe microscopies, including atomic force microscopes (AFMs) and scanning tunneling microscopes. Patterned devices on the scale down to a few tens of nanometers in all 3-D are now possible, leading to increased focus on connections, interfaces, and dimensionality of the SUT.

\section{A. Ballistic Versus Diffusive}

The 2-D materials showing ballistic transport at room temperature over micrometer length scales are now well known. This compromises the concept of the universal property of resistivity of a material, i.e., it can become no longer appropriate to describe a material as having a bulk single-average dissipative property. Instead the interface properties between the material and the measuring system become critical to the observed electrical dissipation. Of course also the anisotropy of 2-D and 1-D materials must be considered in general, although this is not entirely a new phenomenon to metrology. These effects are not only impacting resistance measurement but also the imaginary component of conductivity (i.e., permittivity) in nanoscale confined spaces. It has been recently reported that the permittivity of water (in the bulk as high as 80 due to its strongly polar molecular structure) is reduced to around 2 when the water is confined between two 2-D surfaces with separation of not more than 1-2 nm (or some 


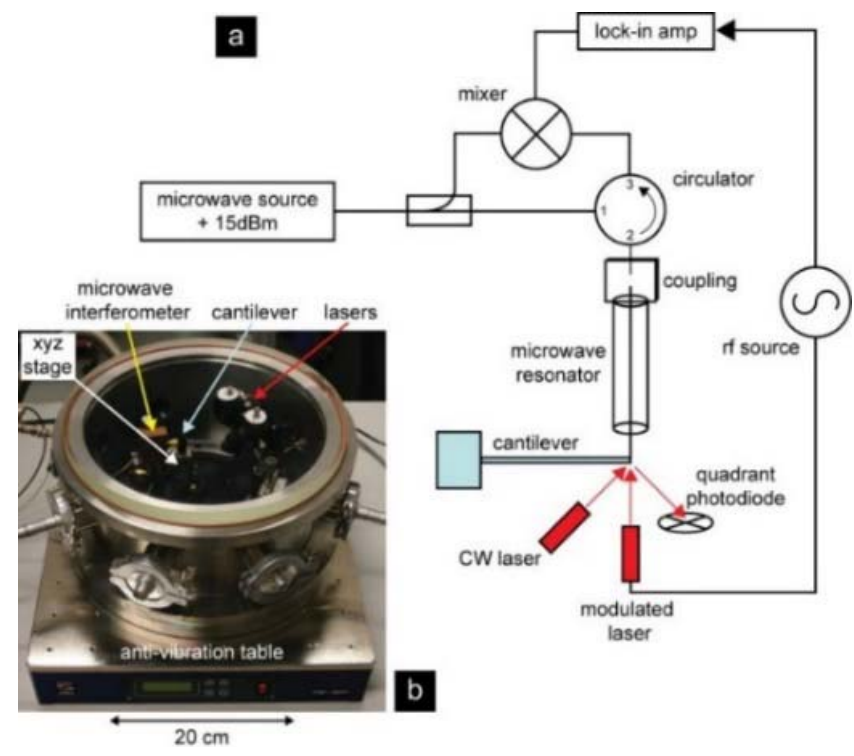

Fig. 1. (a) Schematic of NPL scanned microwave microscope circuit for homodyne detection of surface impedance. (b) Photograph of scanning and readout system inside vacuum chamber with glass lid [9].

three layers of water molecules. Fumagalli et al. [6] suggest that these and future results for this type of system will be important for better understanding of long-range interactions in biological systems, including stability of macromolecules such as DNA and proteins, and of the electric double layer, critical in electrochemistry and energy storage. It is clear that some new concepts as well as measurement techniques are required to take electrical metrology deep into the nanoscale.

\section{Electrical Measurements ON REDUCED DIMENSIONS}

The complexity of the requirements of nanoscale electromagnetic measurements is exemplified by the needs of the new 2-D material graphene. As the first self-supporting single atomic layer material, graphene has high electrical, thermal conductivity and its interfaces with external circuits are problematic. In addition, the conductivity and mobility of this semimetal are both easily influenced by an electric field acting perpendicular to the graphene surface. Many techniques have been developed to deal with these issues but here we discuss one important example [7] which overcomes some of these difficulties, a near-field noncontacting SMM (NSMM).

\section{A. Scanning Microwave Microscopy}

The paradoxical ability to use microwaves (typical freespace wavelength $\sim 1 \mathrm{~cm}$ ) to image on the nanoscale requires the use of the strongly localized evanescent microwave near-field existing in the neighborhood of a sharply pointed conductor driven by a microwave signal [7], [8]. An additional advantage of using microwaves is that a high $Q$ resonator (unavailable at lower frequencies) can be incorporated with the sharp tip, to give enhanced gain. A home-built National Physical Laboratory (NPL) NSMM (Fig. 1) is based on a quarter-wavelength coaxial resonator, filled with a high permittivity $\left(\varepsilon_{r} \sim 20\right)$ dielectric material, having a lowest

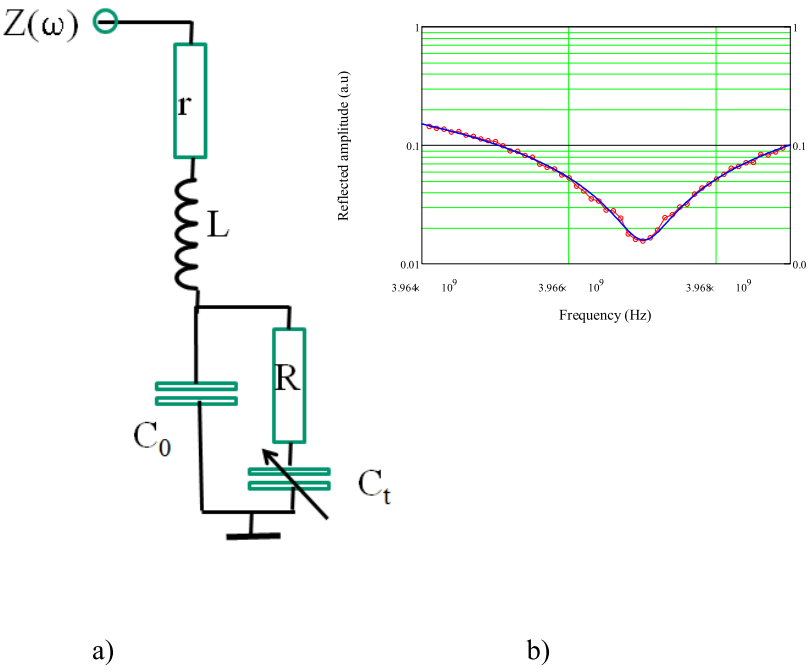

Fig. 2. (a) Schematic of lumped component circuit for the dielectric resonator coupled to a conducting thin-film substrate. (b) Theoretical fit using this model equation to the experimental reflection dip of the dielectric microwave resonator.

mode resonant frequency of $4 \mathrm{GHz}$ and length of only $4 \mathrm{~mm}$ with radius $1.25 \mathrm{~mm}$ [9], [10], allowing an unloaded $Q$ value of 700. A short length of sharply tapered tungsten wire (tip radius of curvature $\sim 1 \mu \mathrm{m}$ ) is attached to the open end of the center conductor. The microwave field from the cavity extends into this region and is highly enhanced at the tip, on account of the sharp radius of curvature. The near-field region provides an external contribution to the total stored energy in the coaxial resonator. When a conducting or dielectric element moves in this high field region, the field distribution will be changed, and as a result, the microwave resonator properties will be slightly altered, with a response leading to shifts in both its resonant frequency and $Q$.

To interrogate the resonator, a modified homodyne detection system is shown in Fig. 1. A microwave synthesizer (output power up to $+21 \mathrm{dBm}$ ) provides a signal tunable through the resonant frequency of the dielectric resonator. This may be amplitude or frequency modulated by an external signal supplied by a lower frequency synthesized source. The microwave output passes through a splitter, allowing a fraction of the power to be tapped off, while the remaining power is fed to a circulator. The dielectric coaxial resonator being connected to the adjacent port of the circulator, and the reflected signal from the resonator is returned through the third circulator port to the RF input of a microwave mixer, with the tapped off component of the microwave power acting as the local oscillator input.

To relate the observed change in the reflected microwave signal to the complex conductivity (or equivalently the 2-D surface impedance) of the sample underlying the microwave probe, we have found that a lumped circuit model is an effective means to characterize these properties. Fig. 2 shows the equivalent circuit for the system, where the coaxial resonator is characterized by a capacitance $C_{0}$ and an inductance $L_{0}$ which can be calculated from the known geometry of the resonator together with the permittivity of its dielectric. 
The resonant frequency is fitted to the observed experimental value when far from any dielectric or conducting surface.

\section{B. Quantitative Analysis}

In order to quantify these electrical measurements, it is necessary to model the response of the near-field microwave resonator to change in the electromagnetic environment close to the tip region. The first approach to this problem treats the coaxial dielectric resonator as a lumped $L C$ circuit with the tip to substrate interaction introducing an additional complex impedance which is spatially dependent on the scan position. The known dimensions and materials of the coaxial quarter-wave resonator provide sufficient accuracy to calculate $L$ and $C_{0}$. The unloaded $Q$ value of the resonator allows $r$ to be calculated. Then, a simple lumped circuit model can provide the predicted influence of the additional contributions $C_{t}$ and $R$ which come from the tip-to-substrate interaction. Note that as this is a noncontact scanning system, the contact resistance between tip and sample is not involved in $R$ which represents the spreading resistance of microwave current flow into the surface film. This is not directly the sheet resistance of the material. To convert the values derived for $R$ from the lumped component model, it is necessary to model the electric field and current distribution within the thin film and the underlying substrate. We are in the process of developing a 3-D finite-element model which will allow simultaneous deconvolution of the sheet resistance and complex conductivity of the underlying substrate from this data.

In the following sections, we describe some developing applications of the SMM, focusing on measuring materials properties and characterization of nano-electromechanical resonators.

\section{Graphene Conductivity Measurements}

The system has been used to make 2-D scans across both the dielectric and conducting thin films and is able to resolve changes in the local properties down to a length scale of around $1 \mu \mathrm{m}$. We have recently used this to image variations in local sheet resistance of transferred chemical vapor deposition (CVD) graphene films (Fig. 3). The upper plot shows how the width of the reflected resonance line varies across the scan, showing a qualitative variation by a factor of 2 in comparing the linewidth with the unperturbed resonance when the tip is far from any surface. The lower scan shows how the center frequency of the response varies with scan position.

This qualitative information proves useful since the linewidth gives information about local graphene conductivity and its spatial variation, whereas the frequency shift reflects the local variations in permittivity. The former tells us about quality or layer number of the graphene, while the latter indicates the presence of some dielectric material polymethyl methacrylate left over from the graphene transfer process. However, neither of these measurements is immediately translated into quantitative values for conductivity or dielectric thickness. In Section VI, we discuss how future developments can be expected to rectify this situation and provide quantitative and ultimately traceable measurements with this system. a)

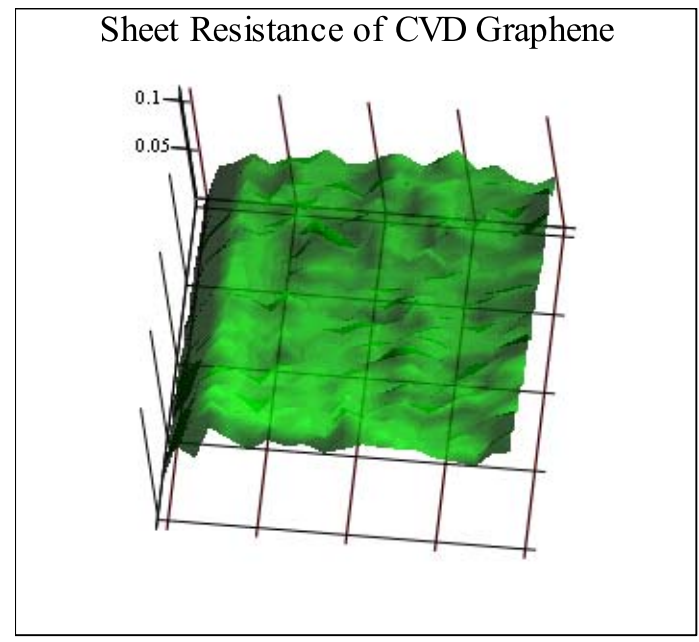

b)

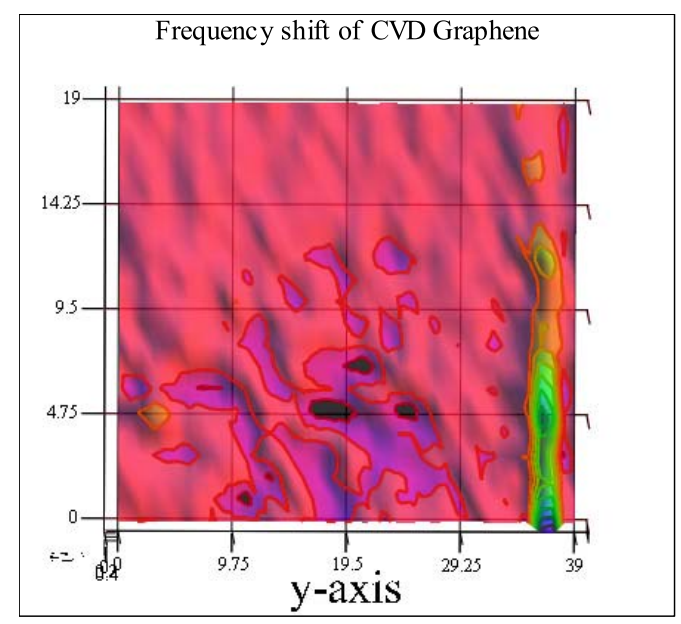

Fig. 3. (a) 2-D scan of sheet resistance shifts across a $0.2 \times 0.4 \mathrm{~mm}^{2}$ region of CVD graphene deposited on quartz substrate. (b) Scan over the same area showing the microwave resonator frequency shift. This is proportional to the local variation of tip to substrate capacitance.

\section{NEMS Resonators}

The flexibility of the NSMM system is further demonstrated by its ability to excite and readout mechanical resonators, down to the nanoscale. As we have noted, the convolution of physical size, real and imaginary parts of electric conductivity, and relative permeability of any SUT make accurate measurements of nanoscale electromagnetic properties complex. Additional complexity arises if NEMSs are also considered. For example, the radiation pressure at the tip of the NSMM exerts a measurable force on a conducting sample and if this is free to move, this movement will change the reflected microwave signal from the probe, making it hard to distinguish a spatial from an electrical variation. However, the interaction between tip and sample can be put to good use since by modulating the amplitude of the microwave signal at a variable frequency any mechanical resonances in the freely suspended sample may be detected.

The microwave field exerts a force on the mechanical resonator (proportional to the electric field) since the combination of sharp tip and mechanical resonator can be treated as electrodes of a capacitor whose value changes with relative movement. In order to apply a time-varying force, 


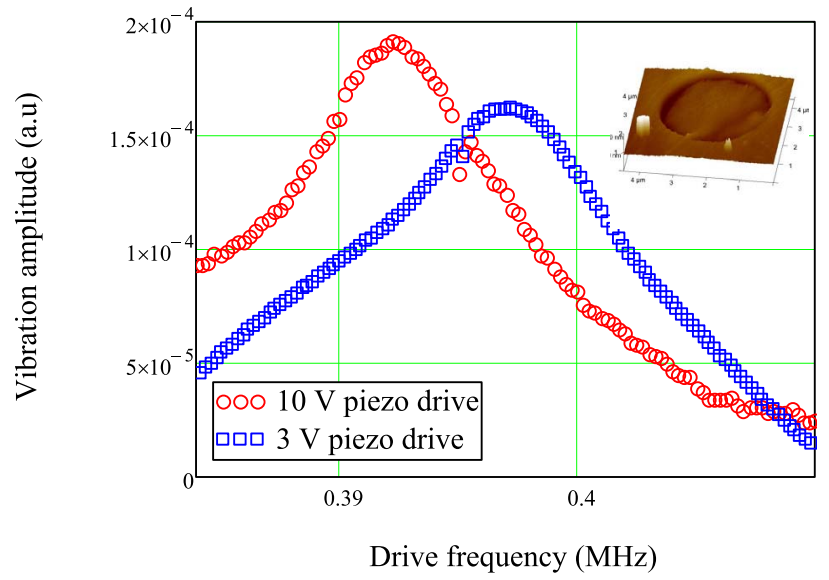

Fig. 4. Monolayer CVD graphene drum resonator ( $3 \mu \mathrm{m}$ diameter) response to two levels of piezodrive, showing a softening of the resonance as the drive level is increased [12]. Inset: AFM image of the $3-\mu \mathrm{m}$-diameter well.

the amplitude of the microwave field must be modulated. In this way motion of microscale and nanoscale cantilevers has been imaged, measuring the shape of the displacement along the diameter of a drum or length of a cantilever (see Section IV). The technique can detect nonlinear behavior including Duffing-type response and has been used extensively at NPL to investigate graphene drum resonators.

\section{E. Graphene Drum Resonators}

The unique properties of graphene make it highly promising for NEMS applications. The 2-D material is both highly crystalline and very strong, with high breaking strain and exceptional Young's modulus, low mechanical dissipation, implying high mechanical $Q$ values, combined with low areal density. A wide range of sensor applications at the single particle and quantum end of the spectrum are anticipated. Graphene drum membrane resonators present large capture area (being 2-D) as well as simple excitation, readout, and functionalization.

We have carried out a number of measurements on CVD monolayer graphene transferred onto Si substrates which have been previously lithographically patterned with an array of "wells" of some micrometers deep (inset of Fig. 4). The Si chip with graphene surface layer is mounted on a piezodisplacer to which a variable frequency ac drive voltage is applied. The tip of the microwave microscope is placed over one of the drums, and the reflected microwave signal is measured as the piezodrive frequency is swept. The reflected microwave signal is fed to a mixer whose local oscillator input is provided by the microwave synthesizer, tuned to be at the point on the reflection dip which is most sensitive to changes in frequency and $Q$. In this way, the mechanical resonance of the suspended graphene drum can be measured. We have carried out measurements on a range of drum diameters from 3 to $30 \mu \mathrm{m}$ observing fundamental resonant frequencies ranging from $28 \mathrm{kHz}$ (for the largest drums) up to $350 \mathrm{kHz}$ for the smallest size. Unlike some authors (see [11]), we observe resonant frequencies corresponding to unstrained graphene films, perhaps reflecting the nature of the substrate used and or the transfer process [12]. As shown by

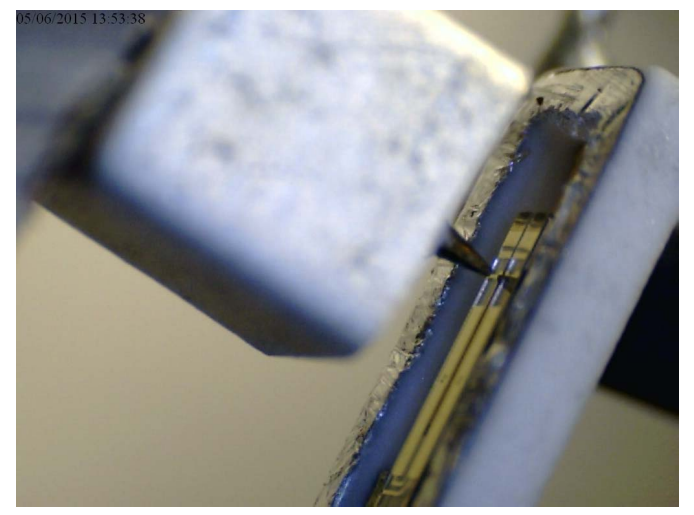

Fig. 5. View of the near-field microwave microscope tip positioned close to oscillating arm of tuning fork cantilever.

the Raman spectroscopy, the CVD graphene is greater than $90 \%$ monolayer. The $Q$ values observed at room temperature in air range from 50 up to 500, and all drum resonances show a softening of the resonant frequency as the piezodrive level is increased (a negative Duffing coefficient), possibly indicating relatively poor adhesion between graphene and substrate.

\section{AC Displacement Calibration}

The near-field microwave method is not only capable of measuring the complex conductivity variations across a sample but also capable of measuring the resonant frequency of small mechanical resonators. It may also be used to image the spatial shape of excited mechanical resonators, such as cantilevers or drum resonators. First, a series of microwave reflection dips are taken with successively closer separation between tip and fork. Then, one of these positions is selected, where the frequency variation with approach distance along the $x$-axis is high. As the tip is moved over a calibrated $x$-axis distance around the chosen tip-cantilever position, the gain $d V_{0} / d x$ is measured. The microwave frequency $f$ is then fixed at a value, where the gain $d V_{0} / d f$ is greatest. Then, driving the cantilever at one of its mechanical resonances, the near-field tip is scanned along the length of the oscillating cantilever while the output of the microwave mixer is measured. The output $V_{\mathrm{ac}}$ at the mechanical drive frequency is extracted from the IF signal from the mixer by feeding it to a lock-in amplifier referenced by the cantilever drive signal. Then, the $x$-axis amplitude of the fork oscillation is just $x_{\mathrm{ac}}$ where

$$
x_{\mathrm{ac}}=\frac{V_{\mathrm{ac}}}{d V_{0} / d x} .
$$

Fig. 5 shows the near-field microscope imaging the resonant displacement of one arm of a tuning fork, driven at a resonant frequency of $10.8 \mathrm{kHz}$ in its lowest out-of-plane flexural mode. This was selected rather than one of the in-plane modes at around $32 \mathrm{kHz}$ as it was easier to bring the scanning tip close to the tuning fork from this direction. Fig. 6 shows the experimental data as the tip is scanned along the length of the tuning fork, compared with a fit to the theoretical form for a simple homogenous cantilever clamped at one end. The shape derived experimentally is seen to be in good agreement with the theoretical shape over the length of the fork which is uniform. Departures are observed at the free end of the 


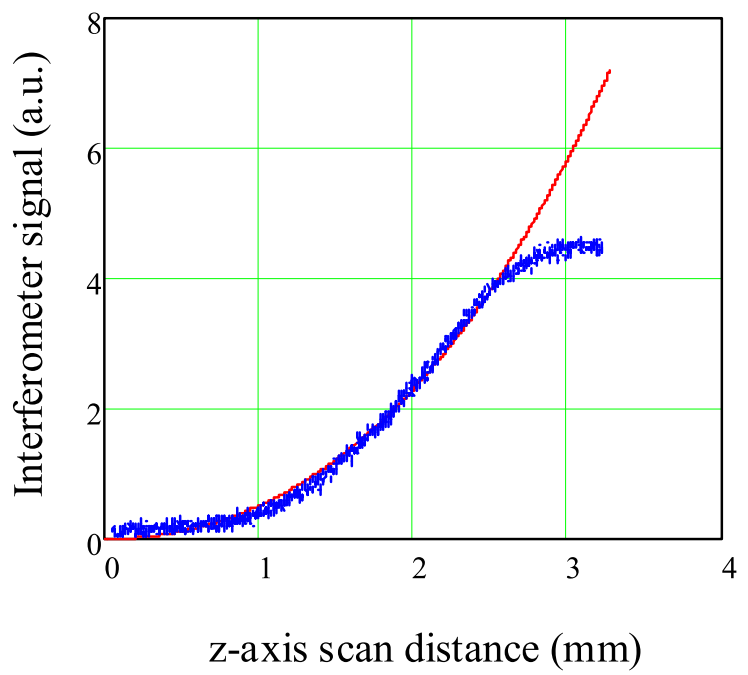

Fig. 6. Measured oscillation amplitude (blue points) of the driven cantilever as a function from the fixed end of the fork $(z=0)$ compared with the theoretical shape for a single clamped cantilever (solid line).

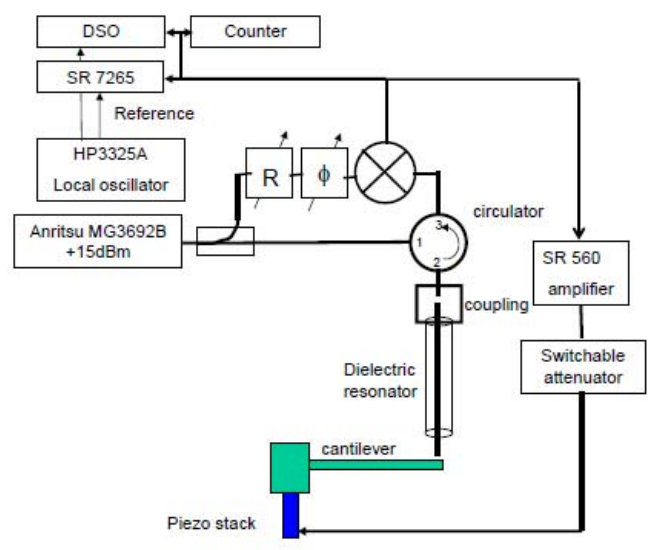

Fig. 7. Circuit diagram for microwave near-field self-oscillating cantilever drive system.

fork where its homogeneity changes due to additional metallic electrodes.

\section{Self-Oscillating Mechanical Resonator}

Although piezoexcitation has a number of limitations it can enable a self-oscillating mechanical resonator system. Simple modifications to the homodyne detection system (shown in Fig. 7, compared with the original in Fig. 1) allow the IF output from the microwave mixer to be fed back via a variable attenuator to a piezostack on which the mechanical resonator is mounted. After an elapsed time of just a few inverse linewidths of the mechanical resonator, the thermal noise motion of this cantilever is amplified by the microwave detection system and fed back with positive gain through the piezoelement. This causes the cantilever to burst into oscillation at a stable resonant frequency which can be measured with a frequency counter. Alternatively, the mixer output can be mixed with an RF local oscillator, signal being fed to a lock-in amplifier whose output will measure the time-dependent phase between mechanical resonator and local oscillator. Analysis of



Fig. 8. Phase noise as a function of sampling frequency for a self-oscillating mechanical cantilever.

this time-dependent phase wander provides a measure of the stability of the mechanical oscillator. A plot of the phase noise as a function of the sampling frequency is shown in Fig. 8.

The self-oscillator demonstrates the high sensitivity of a mechanical resonator as a small force detector. The cantilever, oscillating at a resonant frequency of $6 \mathrm{kHz}$, was capable of detecting applied force changes of less than $30 \mathrm{fN}$, applied to the cantilever by a very low-power laser beam [13].

\section{TRACEABILITY}

The near-field microwave microscope is a very versatile tool, capable of detecting electrical properties of metal or semiconducting thin films on insulating or semiconducting substrates. In addition, its ability to excite and read out the mechanical oscillations of NEMS and MEMS provides high sensitivity measurements of applied force, displacement, and mass addition. The metrological impact of these capabilities will be much enhanced if traceable methods can be developed and applied to this technology. Current developments at NPL involve converting the scanned raw microwave resonance data to conductivity and permittivity of the underlying material. Simple circuit-based modeling provides good measures of relative changes in both the real and imaginary components of SUT impedance. However, scaling these variations to material parameters such as conductivity or permittivity is more complex. One approach uses the mean parameter values measured with a far-field dielectric resonator. A second system employs the observed frequency shift in the microwave resonance as a servo signal to control the distance between tip and SUT. This has been used extensively [14] but is not appropriate if the permittivity of the surface is also subjected to spatial variation. A more precise approach, currently under test, uses standard thin-film conducting and insulating samples. In the longer term simultaneous monitoring of multimodes of the microwave, dielectric resonator may provide higher confidence levels in the deconvolution of spatial and electrical property variations. Note this paper is an extended version of the CPEM 2018 Proceedings Digest paper of the same title [15]. 


\section{REFERENCES}

[1] M. J. T. Milton, R. Davis, and N. Fletcher, "Towards a new SI: A review of progress made since 2011," Metrologia, vol. 51, no. 3, pp. R21-R30, 2014.

[2] J. Gallop, "Fundamental metrology in the future: Measuring the single quantum," Eur. Phys. J. Special Topics, vol. 172, pp. 399-408, 2009.

[3] C. A. Regal, J. D. Teufel, and K. W. Lehnert, "Measuring nanomechanical motion with a microwave cavity interferometer," Nature Phys., vol. 4, pp. 555-560, May 2008.

[4] K. L. Ekinci and M. L. Roukes, "Nanoelectromechanical systems," Rev. Sci. Instrum., vol. 76, no. 6, p. 061101, 2005.

[5] M. Li, H. X. Tang, and M. L. Roukes, "Ultra-sensitive NEMS-based cantilevers for sensing, scanned probe and very high-frequency applications," Nature Nanotechnol., vol. 2, pp. 114-120, Feb. 2007.

[6] L. Fumagalli et al., "Anomalously low dielectric constant of confined water," Science, vol. 360, no. 6395, pp. 1339-1342, 2018.

[7] A. Imtiaz, T. M. Wallis, and P. Kabos, "Near-field scanning microwave microscopy: An emerging research tool for nanoscale metrology," IEEE Microw. Mag., vol. 15, no. 1, pp. 52-64, Jan. 2014.

[8] T. Faust, P. Krenn, S. Manus, J. P. Kotthaus, and E. M. Weig, "Microwave cavity-enhanced transduction for plug and play nanomechanics at room temperature," Nature Commun., vol. 3, Feb. 2012, Art. no. 728.

[9] L. Hao, J. C. Gallop, and D. Cox, "Excitation, detection, and passive cooling of a micromechanical cantilever using near-field of a microwave resonator," Appl. Phys. Lett., vol. 95, no. 11, p. 113501, 2009.

[10] L. Hao, S. Goniszewski, J. Gallop, and J. Chen, "Development of nearfield microwave methods for NEMS resonators," in Proc. IEEE Int. Conf. Nanotechnol., Aug. 2013, pp. 379-383.

[11] C. Chen and J. Hone, "Graphene nanoelectromechanical systems," Proc. IEEE, vol. 101, no. 7, pp. 1766-1779, Jul. 2013.

[12] S. Goniszewski, O. Shaforost, N. Klein, J. Gallop, L. Hao, and D. Cox, "Frequency readout of nanomechanical graphene drums via a microwave resonator coupling method," in Proc. 44th Eur. Microw. Conf., 2014, pp. 363-366.

[13] L. Hao, J. Gallop, M. Stewart, K. Lees, and J. Chen, "Multi-functional MEMS/NEMS for nanometrology applications," in Proc. 13th IEEE Int. Conf. Nanotechnol., Beijing, China, Aug. 2013, pp. 1119-1124.

[14] S. E. de Graaf, A. V. Danilov, A. Adamyan, and S. E. Kubatkin, "A nearfield scanning microwave microscope based on a superconducting resonator for low power measurements," Rev. Sci. Instrum., vol. 84, no. 2, p. 023706, 2013.

[15] L. Hao, J. Gallop, and J. Chen, "Electromagnetic metrology for NEMS," in Proc. CPEM, Paris, France, 2018, pp. 1-2.

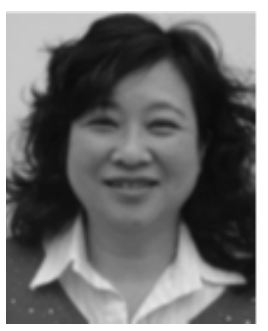

Ling Hao received the B.Sc. degree in general physics and the M.Sc. degree in solid-state physics from Beijing Normal University, Beijing, China, in 1984 and 1987, respectively, and the Ph.D. degree from the University of Strathclyde, Glasgow, U.K., in 1995, with a focus on electronic noise in superconducting devices.

Since 1996, she has been a Senior Research Scientist/Principal Research Scientist with the National Physical Laboratory, Teddington, U.K. She is also a Visiting Professor with the Imperial College, London, U.K. She is leading research on applications of nanoscience, superconducting electronics, and microwave technology for precision measurements, aimed at single particle measurements and metrology with nano-Superconducting QUantum Interference Devices (SQUIDs) and nanoelectromechanical system resonators, low-dimensional carbon, including carbon nanotubes, and graphene transport measurements. She has authored more than 150 papers in refereed journals and five book chapters.

Prof. Hao is currently a member of the Superconductivity Committee of the Institute of Physics. She is a fellow of the Institute of Physics, U.K., and a Chartered Physicist.

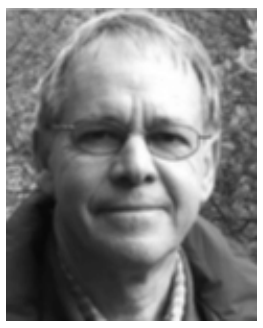

John C. Gallop received the B.A. and D.Phil. degrees in ultralow temperature physics from Oxford University, Oxford, U.K.

He joined the National Physical Laboratory (NPL), Teddington, U.K., in 1969, where he focused on metrology applications of macroscopic quantum aspects of superconductivity (involving fundamental constant determinations, SQUIDs, and highfrequency applications) for a number of years. Later he spent more than a decade on research into the applications of high-temperature superconductivity. He was the Head of the Quantum Detection Group, NPL, till he retired in 2003. He is currently an Emeritus Senior NPL Fellow. His current research interests include developments in nanoscience, including transport properties of graphene and carbon nanotubes, single particle measurements with nano-SQUIDs, submicrometer Hall sensors, and the metrology of nano-electromechanical resonators.

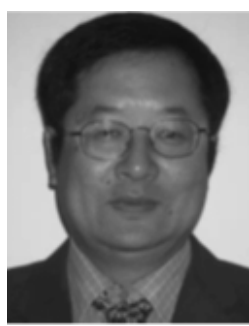

Jie Chen (M'91) received the B.Eng. and M.Sc. degrees from Beihang University, Beijing, China, in 1984 and 1987, respectively, and the D.Phil. degree from the University of York, York, U.K., in 1995 .

He was a Lecturer/Research Associate with the University of Hull, Hull, U.K.; the University of Strathclyde, Glasgow, U.K.; and the University of York. Since 1998, he has been a Lecturer and Senior Lecturer with Brunel University, Uxbridge, U.K. His research book on fault diagnosis for dynamic systems has attracted more than 2700 citations. He has authored more than 60 papers. His current research interests include robust control systems, fault diagnosis, and fault-tolerant control, with emphasis on industrial applications such as aircraft control system and automotive control systems, multiphysics modeling of nanoscale sensing devices, and he is very experienced in applying nonlinear system modeling techniques to thermal and environmental systems.

Dr. Chen is a member of the IFAC Technical Committee SAFEPROCESS. $\mathrm{He}$ is a fellow of the U.K. Institute of Physics. He received the Best Journal Paper Prize from the IET/IEE in 1997. He is an Associate Editor of the International Journal of System Science. 